\title{
La pédagogie de l'enseignement supérieur : une discipline sans frontière
}

\section{Christelle Lison}

\section{(2) OpenEdition \\ 1 Journals}

Édition électronique

URL : http://journals.openedition.org/ripes/1189

DOI : 10.4000/ripes.1189

ISSN : 2076-8427

Éditeur

Association internationale de pédagogie universitaire

Référence électronique

Christelle Lison, "La pédagogie de l'enseignement supérieur : une discipline sans frontière », Revue internationale de pédagogie de l'enseignement supérieur [En ligne], 33(1) | 2017, mis en ligne le 06 mars 2017, consulté le 23 septembre 2020. URL : http://journals.openedition.org/ripes/1189; DOI : https:// doi.org/10.4000/ripes. 1189

Ce document a été généré automatiquement le 23 septembre 2020.

Article L.111-1 du Code de la propriété intellectuelle. 


\title{
La pédagogie de l'enseignement supérieur : une discipline sans frontière
}

\author{
Christelle Lison
}

1 Pour ce premier numéro de l'année 2017, RIPES se veut cosmopolite en accueillant des collègues français, suisses, belges, canadiens et québécois! C'est la démonstration que la pédagogie de l'enseignement supérieur trouve sa place dans de nombreuses institutions francophones. Les sujets sont variés comme on peut le constater dans le présent numéro et mettent en lumière les préoccupations non seulement de certains collègues, mais aussi de certaines institutions. En effet, elles sont aujourd'hui nombreuses à accorder une place de choix à la réflexion pédagogique.

2 Au même moment, dans ce numéro de RIPES, nous constatons que de nombreux collègues «parrainent » les plus jeunes en co-rédigeant avec eux des articles. Nous ne pouvons que nous en réjouir puisque c'est ce travail collaboratif qui donne, en tout cas en partie, du sens à notre travail. C'est le cas de l'article de Marco Guilbault (ancien étudiant) et d'Anabelle Viau-Guay (professeure) de l'Université Laval (Québec) qui tente de faire un état des connaissances (depuis les 15 dernières années) et quelques recommandations sur l'un des sujets les plus discutés du moment en enseignement supérieur, la classe inversée. C'est là un pari ambitieux que de tenter de dégager quelques conclusions empiriques afin d'éclairer les collègues et les institutions souhaitant s'y lancer.

3 Le deuxième article de ce numéro est celui de Christophe Michaut (enseignantchercheur) et de Marine Roche (doctorante) de l'Université de Nantes (France). Ils proposent de discuter de l'influence des usages numériques des étudiants sur leur réussite universitaire, et notamment sur les résultats aux examens. Ils tirent leurs conclusions à partir d'une étude menée auprès de 625 étudiants français inscrits en premier cycle universitaire de Santé, de Sciences et techniques, et de Lettres et Sciences humaines et sociales. Au final, Michaut et Roche constatent que les étudiants 
s'emparent assez peu des outils numériques pour leurs apprentissages et que lorsqu'ils le font, c'est essentiellement dans une visée instrumentale.

4 Traitant aussi du numérique, mais sous l'angle d'un dispositif de ePortolfio, Laurent Verslype, Julie Lecoq, Pascale Corten-Gualtieri et Emmanuelle Druart, de l'Université catholique de Louvain (Louvain-la-Neuve, Belgique), proposent l'évaluation de ce dispositif mis en place par le Département d'histoire de l'art et d'archéologie de l'Université catholique de Louvain. A partir de leurs analyses, ils démontrent que les modalités d'accompagnement de la prise d'autonomie des étudiants ainsi que l'évaluation formative de leur travail au développement de plusieurs compétences génériques essentielles dans la formation universitaire, notamment l'esprit analytique, critique et de synthèse, la capacité à rédiger un texte de niveau universitaire et à argumenter.

5 Faisant un pas de côté, Alexia Stumpf et Paul-André Garessus de la Haute Ecole Pédagogique -BEJUNE (Suisse) nous offrent un article portant sur l'évaluation, dans une posture formative, de la qualité de la formation dans une institution d'enseignement supérieur et notamment une démarche d'analyse d'outils évaluatifs dans le contexte d'une haute école pédagogique suisse. En présentant la démarche de manière générale, ils visent sa transférabilité à divers contextes de formation supérieure tout en esquissant quelques pistes d'évolution possibles afin de contrer les limites qu'ils ont identifiées.

6 Le cinquième article, celui d'Eva Lemaire, de l'University of Alberta (Canada), nous emmène dans un univers complètement différent, celui de 14 étudiants en éducation canadiens, partis enseigner dans une école du Massaï Mara, dans le cadre d'un stage encadré par les organismes humanitaires Free the Children/Me to We. A partir d'entrevues individuelles et de notes d'observations, Lemaire interroge "l'impact» d'une telle expérience en termes d'éducation interculturelle et de citoyenneté globale. L'analyse met en relief des discours ambivalents entre volonté de respecter une culture éducative posée comme Autre, désir d'apporter un changement par l'apport de pratiques éducatives différentes et minoration des variations interculturelles. Cet article souligne également l'importance de la formation à l'interculturel, au-delà de l'expérientiel.

7 Le sixième article de ce numéro RIPES est à nouveau issu d'une collaboration entre une étudiante et une enseignante toutes deux du Service d'Orthopédagogie Clinique de l'Université de Mons (Belgique). Dans celui-ci, Hélène Geurts et Marie-Claire Haelewyck partent du constat que les étudiants inscrits en psychologie s'intéressent peu aux options traitant de l'avancée en âge, malgré la croissance des opportunités dans ce domaine. Par le biais d'un questionnaire, les deux auteurs ont interrogé les étudiants sur leurs représentations à l'égard de la vieillesse. L'analyse des résultats les amène à penser que la faible orientation vers les professions gérontologiques n'est pas liée aux représentations exclusivement négatives de l'avancée en âge, mais réfère également au manque de compétences, de légitimité perçue par ces jeunes en quête de repères et de valorisation. Ce sont donc des pistes de travail pour les enseignants évoluant dans ces formations.

8 Finalement, pour ce premier numéro de l'année 2017, Dieudonné Leclercq, de l'Université de Liège (Belgique), nous propose la recension d'un ouvrage de Miguel Souto Lopez, intitulé Acquis d'apprentissage et enseignement supérieur. Celui-ci analyse les processus qui ont conduit les établissements d'enseignement supérieur à définir des 
acquis d'apprentissage pour chacun de leurs programmes, en s'intéressant plus particulièrement à l'Université catholique de Louvain, à l'Université Libre de Bruxelles et à l'Université de Liège.

9 Christelle Lison, Sherbrooke, mars 2017

\section{AUTEUR}

CHRISTELLE LISON

Sherbrooke 